\title{
Osmophoresis of a Spherical Vesicle in a Circular Cylindrical Pore
}

\author{
Huan J. Keh and Yun S. Hsu \\ Dept. of Chemical Engineering, National Taiwan University, Taipei 106-17, Taiwan, Republic of China \\ DOI 10.1002/aic.10510 \\ Published online July 7, 2005 in Wiley InterScience (www.interscience.wiley.com).
}

\begin{abstract}
The problem of the osmophoretic motion of a spherical vesicle along the centerline of a circular cylindrical pore is studied theoretically in the quasi-steady limit of negligible Reynolds and Peclet numbers. The imposed solute concentration gradient is uniform and parallel to the pore wall, which may be either impermeable to the solute molecules or prescribed with the far-field concentration distribution. The presence of the pore wall causes two basic effects on the vesicle velocity: (1) the local concentration gradients on the vesicle surface are altered by the wall, thereby speeding up or slowing down the vesicle; (2) the wall enhances the viscous interaction effect on the moving vesicle. To solve the equations of conservation of mass and momentum, the general solutions are constructed from the fundamental solutions in both cylindrical and spherical coordinates. The boundary conditions are enforced first at the pore wall by the Fourier transforms and then on the vesicle surface by a collocation technique. Numerical results for the osmophoretic velocity of the vesicle, relative to that under identical conditions in an unbounded solution, are presented for various values of the relevant properties of the vesicle as well as the relative separation distance between the vesicle and the pore wall. The collocation results agree well with the approximate analytical solution obtained by using a method of reflections. The presence of the wall will enhance the vesicle velocity, although its dependency on the ratio of vesicle-to-pore radii is not necessarily to be monotonic. In general, the boundary effect on osmophoresis is quite significant. () 2005 American Institute of Chemical Engineers AIChE J, 51: 2628-2639, 2005
\end{abstract}

Keywords: osmophoresis, semipermeable vesicle, circular cylindrical pore, boundary effect, fluid mechanics

\section{Introduction}

When two solutions differing in solute concentration are separated by a semipermeable membrane (that permits the passage of solvent but not of solute), the solvent at the side of lower concentration tends to pass through the membrane into the solution of higher concentration. This is the phenomenon of osmosis; it still occurs to a certain extent when the solute molecules can cross the membrane but undergo more resistance in doing so than the solvent molecules. The osmotic flow of

Correspondence concerning this article should be addressed to H. J. Keh at huan@ntu.edu.tw.

(C) 2005 American Institute of Chemical Engineers solvent can be prevented by applying a pressure to the solution of higher concentration, which is greater than the pressure on the solution at the other side by an amount equal to $\sigma \Delta \Pi$, where $\Delta \Pi$ is the difference in osmotic pressure between the two solutions and $\sigma$ is a reflection coefficient characterizing the degree to which the solute molecules are rejected from the membrane. For a semipermeable membrane, $\sigma=1$; for a nonselective membrane, $\sigma=0$. The osmotic pressure $\Pi$ is linearly related to the solute concentration $C$ by the van't Hoff law ( $\Pi=C R T$, where $R$ is the gas constant and $T$ is the absolute temperature) for an ideal solution (with very low solute concentration).

As a vesicle, which is a body of fluid surrounded by a semipermeable membrane, is placed in a solution possessing a 
solute concentration gradient, one pole of the vesicle is exposed to a higher solute concentration (and thus a higher osmotic pressure) than the opposite pole. The osmotic driving force causes the solvent to cross the vesicle's membrane from inside to outside at the high concentration pole, and from outside to inside at the low concentration pole. The vesicle thus functions as a microengine, sucking fluid into it on one side and ejecting fluid on the other, thereby advancing toward regions of low concentration (that is, in the direction of the solute diffusion current). This phenomenon of vesicle movement is termed osmophoresis $^{1-3}$ and could play some role in the motility of biological vesicles and cells.

Anderson $^{3,4}$ analyzed in considerable detail the osmophoretic motion of a spherical or ellipsoidal vesicle with a thin, rigid membrane. He calculated the drift velocity of the vesicle placed in an unbounded fluid with a prescribed linear solute concentration distribution $C_{\infty}(\mathbf{x})$ far away from the vesicle for a quite general case. In most physically realistic systems, the velocity $\mathbf{U}_{0}$ of a semipermeable spherical vesicle of radius $a$ is related to the uniform concentration gradient $\nabla C_{\infty}$ by the expression

$$
\mathbf{U}_{0}=-A \nabla C_{\infty}
$$

where the vesicle's mobility

$$
A=\frac{a L_{\mathrm{p}} R T}{2+2 \bar{\kappa}+\kappa}
$$

with dimensionless parameters

$$
\begin{gathered}
\kappa=a L_{\mathrm{p}} R T \frac{C_{0}}{D} \\
\bar{\kappa}=a L_{\mathrm{p}} R T \frac{\bar{C}}{\bar{D}}
\end{gathered}
$$

In the above equations, $L_{\mathrm{p}}$ is the hydraulic coefficient, which is a constant for a given membrane and solvent (equal to the superficial fluid velocity divided by the normal stress difference across the membrane); $\bar{D}$ and $D$ are the solute diffusion coefficients inside and outside the vesicle, respectively; $\bar{C}$ is the average internal concentration of solute; and $C_{0}$ denotes the value of $C_{\infty}$ at the position of the vesicle center. The van't Hoff law was used in the derivation of Eq. 1 ; if $\Pi$ is not a linear function of $C$ at fixed temperature, then $R T$ must be replaced by $\partial \Pi / \partial C$, evaluated at $C_{0}$ in Eqs. $1 \mathrm{~b}$ and $2 \mathrm{a}$ and at $\bar{C}$ in Eq. $2 \mathrm{~b}$. Typical values in aqueous solutions for the parameters in Eq. 1 are $L_{\mathrm{p}}=10^{-8} \mathrm{~m}^{2} \mathrm{~s} / \mathrm{kg},\left|\nabla C_{\infty}\right|=10^{5} \mathrm{~mol} / \mathrm{m}^{4}$, and $\kappa($ or $\bar{\kappa})$ of order unity (obviously, depending on the concentrations and diffusivities of the solutes and the size of the vesicle). Equation 1 shows that the vesicle always moves toward regions of lower $C_{\infty}$, irrespective of the relative values of $C_{0}$ and $\bar{C}$. Increases in the value of parameter $\kappa$ or $\bar{\kappa}$ have a retarding effect on the vesicle velocity. A recent experimental work reported that model lipid (dimyristoyl phosphatidylcholine) vesicles of a $10-\mu \mathrm{m}$ radius in a sucrose or salt concentration gradient of $10^{4}$ $\mathrm{mol} / \mathrm{m}^{4}$ (the lipid bilayers permit passage of water molecules but not of solute molecules) have a drift velocity of a few micrometers per second, ${ }^{5}$ which is slightly greater than, but still close to, that predicted by Eq. 1 .

Equation 1 serves only for external fluids that extend to infinity in all directions. In real situations of osmophoresis, however, vesicles are not isolated and will move in the presence of neighboring boundaries. Using a method of reflections, Anderson $^{6}$ analytically obtained the migration velocity of a spherical vesicle undergoing osmophoresis along the axis of a long circular pore with an impermeable wall for the special case of $\kappa=\bar{\kappa}=0$. His result indicates that the vesicle velocity increases monotonically as the ratio of vesicle-to-pore radii increases. This behavior, which is opposite to intuition and occurs because the flow of solvent accompanying the osmophoretic vesicle is opposite to the direction of vesicle movement, was also observed experimentally by Berg and Turner ${ }^{7}$ for the chemotaxis of Escherichia coli and mutants in 10- and 50- $\mu \mathrm{m}$ - diameter capillary tubes. On the other hand, the osmophoretic motion of a spherical vesicle in an arbitrary direction with respect to a plane wall was examined by Keh and Yang8 through an exact representation in spherical bipolar coordinates, whereas the motion parallel to two plane walls at an arbitrary position between them was investigated by Chen and $\mathrm{Keh}^{9}$ using a boundary collocation method. Numerical results of wall correction to Eq. 1 for the vesicle velocity were presented for various values of the relative separation distances and parameters $\kappa$ and $\bar{\kappa}$. Although the osmophoretic mobility of the vesicle was also found to increase as the vesicle approaches the plane wall for the general cases considered in these works, the presence of a lateral wall can reduce the vesicle mobility in some situations.

The purpose of the present investigation is to obtain exact numerical solutions and approximate analytical solutions for the osmophoretic motion of an arbitrary spherical vesicle with a thin, rigid membrane along the centerline of a long circular cylindrical pore. The pore wall may be either impermeable to the solute species or prescribed with the linear far-field solute concentration distribution. The effects of fluid inertia as well as solute convection are neglected. As will be shown from the method-of-reflection analysis in Appendix A, for the case of a vesicle with $\kappa \gg 1+\bar{\kappa}$ undergoing osmophoresis near an impermeable pore wall or of a vesicle with $\kappa \ll 1+\bar{\kappa}$ undergoing osmophoresis near a pore wall prescribed with the far-field concentration distribution, the solute diffusion around the vesicle will generate smaller concentration gradients along the vesicle surface relative to those in an infinite medium. These concentration gradients reduce the osmophoretic velocity, although it will be enhanced by the viscous interaction of the migrating vesicle with the wall. Both effects of this solutal retardation and the hydrodynamic enhancement increase as the ratio of vesicle-to-pore radii increases. A main object of this work is to determine which effect is overriding at various vesicle-wall gap widths.

\section{Analysis}

We consider the axisymmetric osmophoresis of a spherical vesicle of radius $a$ surrounded by a thin, rigid, semipermeable membrane along the axis of a long circular cylindrical pore of radius $b$, as shown in Figure 1 . Here $(\rho, \phi, z)$ and $(r, \theta, \phi)$ denote the cylindrical and spherical coordinate systems, re- 


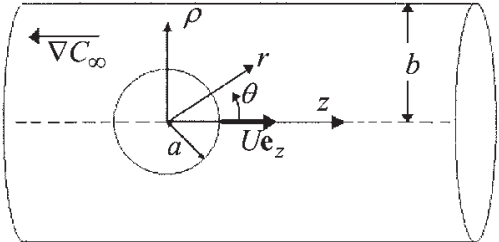

Figure 1. Geometrical sketch for the osmophoresis of a spherical vesicle along the axis of a long circular cylindrical pore.

spectively, and the origin of coordinates is chosen at the vesicle center. A linear concentration field $C_{\infty}(z)$ with a uniform solute gradient $-E_{\infty} \mathbf{e}_{z}\left(=\nabla C_{\infty}\right)$ is imposed in the ambient fluid far away from the vesicle, where $\mathbf{e}_{z}$ is the unit vector in the $z$-direction and $E_{\infty}$ is taken to be positive. The objective is to obtain the correction to Eq. 1 for the vesicle velocity arising from the presence of the pore.

To determine the osmophoretic velocity of the vesicle, it is necessary to ascertain the solute concentration distributions inside and outside the vesicle and the velocity field in the surrounding fluid phase.

\section{Solute concentration distribution}

The osmophoretic motion of a vesicle can be considered quasi-steady if the Peclet and Reynolds numbers are small. The equations of continuity governing the solute concentration distributions for the external and internal fluids are

$$
\nabla^{2} C=0 \quad(r \geq a)
$$

and

$$
\nabla^{2} C_{1}=0 \quad(r \leq a)
$$

respectively. Because the radius of the vesicle is much greater than the thickness of its membrane, $r=a$ can represent both the inner and outer membrane surfaces of the vesicle. Thus, the concentration distribution is subject to the boundary conditions $^{3,8}$

$$
\begin{gathered}
r=a: \quad \frac{\partial C}{\partial r}=\frac{\kappa}{a}\left[C-C_{0}-\left(C_{1}-\bar{C}\right)\right] \\
\frac{\partial C_{1}}{\partial r}=\frac{\bar{\kappa}}{a}\left[C-C_{0}-\left(C_{1}-\bar{C}\right)\right]
\end{gathered}
$$

where the definition of the parameters $\kappa$ and $\bar{\kappa}$ (proportional to $C_{0}$ and $\bar{C}$, respectively) is given by Eq. 2 .

There is no solute to be transferred through the impermeable pore wall and the solute concentration far away from the vesicle approaches the undisturbed quantities. Thus

$$
\begin{gathered}
\rho=b: \frac{\partial C}{\partial \rho}=0 \\
|z| \rightarrow \infty: \quad C \rightarrow C_{\infty} \equiv C_{0}-E_{\infty} z
\end{gathered}
$$

For the case of osmophoretic motion of a vesicle in a pore whose wall is prescribed with a linear concentration profile consistent with the far-field solute distribution, Eq. 5 should be replaced by

$$
\rho=b: \quad C=C_{\infty}
$$

The external concentration distribution $C$, which is governed by the linear Laplace equation and antisymmetric with respect to $z$ after the subtraction of $C_{0}$, can be expressed as the superposition

$$
C=C_{\mathrm{w}}+C_{\mathrm{p}}
$$

Here, $C_{\mathrm{w}}$ is a Fourier-Bessel integral solution of Eq. 3a in cylindrical coordinates, representing the disturbance produced by the pore wall plus the undisturbed concentration field, and is given by ${ }^{10}$

$$
C_{\mathrm{w}}=C_{0}-E_{\infty} z+E_{\infty} \int_{0}^{\infty} R(\omega) I_{0}(\omega \rho) \sin (\omega z) d \omega
$$

where $I_{0}$ is the modified Bessel function of the first kind of order zero and $R(\omega)$ is an unknown function of the variable $\omega$. The second term on the right-hand side of Eq. $8, C_{\mathrm{p}}$, is a solution of Eq. $3 \mathrm{a}$ in spherical coordinates, representing the disturbance generated by the vesicle, and is given by an infinite series in harmonics,

$$
C_{\mathrm{p}}=E_{\infty} \sum_{m=1}^{\infty} T_{m} r^{-m-1} P_{m}(\cos \theta) \quad(m \text { is odd })
$$

where $P_{m}$ is the Legendre polynomial of order $m$ and $T_{m}$ are unknown constants. Note that a solution for $C$ of the form given by Eqs. 8-10 immediately satisfies the boundary condition at infinity in Eq. 6. Because the solute concentration is finite for any position in the interior of the vesicle, the solution to Eq. 3b can be written as

$$
C_{1}=\bar{C}-E_{\infty} z+E_{\infty} \sum_{m=1}^{\infty} \bar{T}_{m} r^{m} P_{m}(\cos \theta) \quad(m \text { is odd })
$$

where $\bar{T}_{m}$ are unknown constants. Only the terms with odd $m$ are included in Eqs. 10 and 11 because both the concentration fields $C-C_{0}$ and $C_{1}-\bar{C}$ are antisymmetric with respect to $z$.

Substitution of the concentration distribution $C$ given by Eqs. 8-10 into the boundary condition 5 or 7 and application of the Fourier sine transform on the variable $z$ lead to a solution for $R(\omega)$ in terms of the coefficients $T_{m}$. After the substitution of this solution into Eqs. 8-10 and use of the integral representations of the modified Bessel functions, $C$ can be expressed as 


$$
C=C_{0}-E_{\infty} z+E_{\infty} \sum_{m=1}^{\infty} T_{m} \delta_{m}^{(1)}(r, \theta) \quad(m \text { is odd })
$$

where the function $\delta_{m}^{(1)}(r, \theta)$ is defined by Eq. B1 in Appendix B. Applying the boundary conditions given by Eq. 4 to Eqs. 11 and 12 yields

$$
\sum_{m=1}^{\infty}\left[T_{m} \bar{\kappa} \delta_{m}^{(1)}(a, \theta)-\bar{T}_{m}(\bar{\kappa}+m) a^{m} P_{m}(\cos \theta)\right]=-a \cos \theta
$$

$$
\begin{array}{r}
\sum_{m=1}^{\infty}\left\{T_{m}\left[\kappa \delta_{m}^{(1)}(a, \theta)-a \delta_{m}^{(2)}(a, \theta)\right]-\bar{T}_{m} \kappa a^{m} P_{m}(\cos \theta)\right\} \\
=-a \cos \theta
\end{array}
$$

where $m$ is odd and the definition of the function $\delta_{m}^{(2)}(r, \theta)$ $\left[=\partial \delta_{m}^{(1)} / \partial r\right]$ is given by Eq. B2.

To satisfy the conditions in Eq. 13 exactly along the entire surface of the vesicle would require the solution of the entire infinite array of unknown constants $T_{m}$ and $\bar{T}_{m}$. However, the collocation method ${ }^{9,11,12}$ enforces the boundary conditions at a finite number of discrete points on the quarter-circular generating arc of the sphere (from $\theta=0$ to $\theta=\pi / 2$, stemming from the symmetry of the system geometry and antisymmetry in the solute concentration fields) and truncates the infinite series in Eqs. 11 and 12 into finite ones. If the spherical boundary is approximated by satisfying the conditions of Eq. 4 at $M$ discrete points on the generating arc, the infinite series in Eqs. 11 and 12 are truncated after $M$ terms, resulting in a system of $2 M$ simultaneous linear algebraic equations in the truncated form of Eq. 13. This matrix equation can be numerically solved to yield the $2 M$ unknown constants $T_{m}$ and $\bar{T}_{m}$ required in the truncated form of Eqs. 11 and 12 for the solute concentration distribution. The accuracy of the truncation technique can be improved to any degree by taking a sufficiently large value of $M$. Naturally, as $M \rightarrow \infty$ the truncation error vanishes and the overall accuracy of the solution depends only on the numerical integration required in evaluating the functions $\delta_{m}^{(1)}$ and $\delta_{m}^{(2)}$ in Eq. 13.

\section{Fluid velocity distribution}

Having obtained the solution for the solute concentration distribution on the vesicle surface that drives the osmophoretic migration, we can now proceed to find the flow field. Because of the low Reynolds number, the fluid motion outside the vesicle is governed by the quasi-steady fourth-order differential equation for viscous axisymmetric creeping flows, as follows

$$
E^{2}\left(E^{2} \Psi\right)=0
$$

in which the Stokes stream function $\Psi$ is related to the velocity components in cylindrical coordinates by

$$
\begin{gathered}
\nu_{\rho}=\frac{1}{\rho} \frac{\partial \Psi}{\partial z} \\
\nu_{z}=-\frac{1}{\rho} \frac{\partial \Psi}{\partial \rho}
\end{gathered}
$$

and the Stokes operator $E^{2}$ has the form

$$
E^{2}=\rho \frac{\partial}{\partial \rho}\left(\frac{1}{\rho} \frac{\partial}{\partial \rho}\right)+\frac{\partial^{2}}{\partial z^{2}}
$$

The boundary conditions for the fluid velocity at the vesicle surface, ${ }^{3,8}$ on the pore wall, and far from the vesicle are

$$
\begin{gathered}
r=a: \quad \mathbf{v}=U \mathbf{e}_{z}+L_{\mathrm{p}} R T\left[C-C_{0}-\left(C_{1}-\bar{C}\right)\right] \mathbf{e}_{r} \\
\rho=b: \quad \mathbf{v}=\mathbf{0} \\
|z| \rightarrow \infty: \quad \mathbf{v}=\mathbf{0}
\end{gathered}
$$

Here, $\mathbf{e}_{r}$ is the unit vector in the $r$ direction and $U$ is the osmophoretic velocity of the vesicle to be determined.

To solve the flow field, we express the stream function, which is symmetric about the plane $z=0$, in the form

$$
\Psi=\Psi_{\mathrm{w}}+\Psi_{\mathrm{p}}
$$

Here $\Psi_{\mathrm{w}}$ is a solution of Eq. 14 in cylindrical coordinates that represents the disturbance produced by the pore wall and is given by a Fourier-Bessel integral ${ }^{10,12}$

$$
\Psi_{\mathrm{w}}=\int_{0}^{\infty}\left[X(\omega) \rho I_{1}(\omega \rho)+Y(\omega) \rho^{2} I_{0}(\omega \rho)\right] \cos (\omega z) d \omega
$$

where $X(\omega)$ and $Y(\omega)$ are unknown functions of $\omega$. The second part of $\Psi$, denoted by $\Psi_{\mathrm{p}}$, is a solution of Eq. 14 in spherical coordinates, representing the disturbance generated by the vesicle, and is given by

$$
\Psi_{\mathrm{p}}=\sum_{n=2}^{\infty}\left(B_{n} r^{-n+1}+D_{n} r^{-n+3}\right) G_{n}^{-1 / 2}(\cos \theta) \quad(n \text { is even })
$$

where $G_{n}^{-1 / 2}$ is the Gegenbauer polynomial of the first kind of order $n$ and degree $-1 / 2 ; B_{n}$ and $D_{n}$ are unknown constants. Note that the boundary condition in Eq. 19 is immediately satisfied by a solution of the form given by Eqs. 20-22.

Substituting the stream function $\Psi$ given by Eqs. $20-22$ into the boundary condition 18 and applying the Fourier cosine transform on the variable $z$ lead to a solution for $X(\omega)$ and $Y(\omega)$ in terms of the coefficients $B_{n}$ and $D_{n}$. After the substitution of this solution into Eqs. 20-22 and use of Eq. 15 and the integral representations of the modified Bessel functions, the fluid velocity components can be expressed as 


$$
\begin{aligned}
& \nu_{\rho}=\sum_{n=2}^{\infty}\left[B_{n} \gamma_{n}^{(1)}(r, \theta)+D_{n} \gamma_{n}^{(2)}(r, \theta)\right] \\
& \nu_{z}=\sum_{n=2}^{\infty}\left[B_{n} \gamma_{n}^{(3)}(r, \theta)+D_{n} \gamma_{n}^{(4)}(r, \theta)\right]
\end{aligned}
$$

where $n$ is even and the definition of functions $\gamma_{n}^{(i)}(r, \theta)$ for $i$ $=1,2,3$, and 4 (which must be performed numerically) is given by Eqs. B3-B6.

The only boundary condition that remains to be satisfied is that on the vesicle surface. Substituting Eqs. 11, 12, and 23 into Eq. 17, one obtains

$$
\begin{aligned}
\sum_{n=2}^{\infty}\left[B_{n} \gamma_{n}^{(1)}(a, \theta)+D_{n} \gamma_{n}^{(2)}(a, \theta)\right] & =L_{\mathrm{p}} R T E_{\infty} \sum_{m=1}^{\infty}\left[T_{m} \delta_{m}^{(1)}(a, \theta)\right. \\
& \left.-\bar{T}_{m} a^{m} P_{m}(\cos \theta)\right] \sin \theta
\end{aligned}
$$

$$
\begin{aligned}
\sum_{n=2}^{\infty}\left[B_{n} \gamma_{n}^{(3)}(a, \theta)\right. & \left.+D_{n} \gamma_{n}^{(4)}(a, \theta)\right]=U+L_{\mathrm{p}} R T E_{\infty} \sum_{m=1}^{\infty}\left[T_{m}\right. \\
& \left.\times \delta_{m}^{(1)}(a, \theta)-\bar{T}_{m} a^{m} P_{m}(\cos \theta)\right] \cos \theta
\end{aligned}
$$

where $m$ is odd, $n$ is even, and the first $2 M$ coefficients $T_{m}$ and $\bar{T}_{m}$ have been determined through the procedure given in the previous subsection.

Equation 24 can be satisfied by making use of the collocation technique presented for the solution of the solute concentration field. At the vesicle surface, Eq. 24 is applied at $N$ discrete points (values of $\theta$ between 0 and $\pi / 2$ ) and the infinite series in Eq. 23 are truncated after $N$ terms. This generates a set of $2 N$ linear algebraic equations for the $2 N$ unknown coefficients $B_{n}$ and $D_{n}$. The fluid velocity field outside the vesicle is completely obtained once these coefficients are solved for a sufficiently large value of $N$.

\section{Derivation of the vesicle velocity}

The hydrodynamic force acting on the spherical vesicle can be determined from ${ }^{13}$

$$
F=4 \pi \eta D_{2}
$$

where $\eta$ is the fluid viscosity. This expression shows that only the lowest-order coefficient $D_{2}$ contributes to the drag force exerted on the vesicle by the external fluid.

Because the vesicle is freely suspended in the surrounding fluid, the net force acting on the vesicle must vanish. Application of this constraint to Eq. 25 gives

$$
D_{2}=0
$$

To determine the osmophoretic velocity $U$ of the vesicle, Eq. 26 and the $2 N$ algebraic equations resulting from Eq. 24 are to be solved simultaneously.

If the vesicle velocity in Eq. 17 is disabled (that is, $U=0$ is set), then the force obtained from Eq. 25 can be taken as the osmophoretic force exerted on the vesicle in the cylindrical pore resulting from the solute concentration gradient $\nabla C_{\infty}$. This force can be expressed as

$$
F=6 \pi \eta a U_{0} F^{*}
$$

where $U_{0}$ is a characteristic velocity (the osmophoretic velocity of the vesicle in the absence of the pore wall) given by Eq. 1 and $F^{*}$ is the normalized magnitude of the osmophoretic force. The value of $F^{*}$ also equals $f^{*} U / U_{0}$, where $f^{*}$ is the dimensionless Stokes resistance coefficient of the vesicle migrating along the axis of the cylindrical pore driven by a body force in the absence of the concentration gradient and $U$ is the osmophoretic velocity of the vesicle obtained from Eq. 26. This dimensionless resistance coefficient $f *$ is the same as that of an impermeable solid sphere of identical radius translating along the axis of the cylindrical pore under a body force field. ${ }^{12,13}$ To see this, the osmophoresis problem can be deconstructed into two subproblems: (1) the vesicle at rest under a solute concentration gradient experiences a force that is exactly $F$ given by Eq. 27, and (2) the vesicle moving at velocity $U$ as a result of a body force experiences a drag force $6 \pi \eta a U f *$, which is also equal to $F$ such that the net force vanishes. Such an analysis clearly dictates that $f^{*}$ stands for the resistance coefficient of the vesicle, not really an impermeable sphere. However, in the absence of a concentration gradient, no radial fluid velocity relative to the vesicle center exists at the vesicle surface as seen from Eq. 17, despite a nonzero $L_{\mathrm{p}}$. This would imply that the drag force on a vesicle under a body force field is equal to that on an impermeable sphere, and solvent permeation across the membrane takes place only in the presence of a concentration gradient.

\section{Results and Discussion}

The numerical results for the osmophoretic motion of a spherical vesicle along the axis of a cylindrical pore, obtained by using the boundary collocation method described in the previous section, is presented in this section. The system of linear algebraic equations to be solved for the coefficients $T_{m}$ and $\bar{T}_{m}$ is constructed from Eq. 13, whereas that for $B_{n}$ and $D_{n}$ is constructed from Eq. 24. All the numerical integrations to calculate the functions $\delta_{m}^{(i)}$ and $\gamma_{n}^{(i)}$ were done by the 180-point Gauss-Laguerre quadrature.

When selecting the points along the quarter-circular generating arc of the spherical vesicle where the boundary conditions are to be exactly satisfied, the first point that should be chosen is $\theta=\pi / 2$, given that this point defines the projected area of the vesicle normal to the direction of motion and controls the gap between the vesicle and the pore wall. In addition, the point $\theta=0$ is also important. However, an examination of the systems of linear algebraic equations in Eqs. 13 and 24 shows that the matrix equations become singular if these points are used. To overcome this difficulty, these points are replaced by closely adjacent points, that is, $\theta=\delta$ and $\pi / 2-\delta .{ }^{12}$ Additional points along the boundary are selected to divide the quartercircular arc of the vesicle into equal segments. The optimum value of $\delta$ is found by considering a succession of the solution in which the boundary conditions are satisfied at the two basic points only, and varying $\delta$. The result reveals that the numerical 
Table 1. Numerical Results of the Normalized Osmophoretic Force $F^{*}$ on a Spherical Vesicle Located on the Axis of a Circular Cylindrical Pore Caused by an Axial Solute Concentration Gradient for the Case of $\kappa=\bar{\kappa}=0$

\begin{tabular}{|c|c|c|c|}
\hline \multirow[b]{2}{*}{$a / b$} & \multicolumn{3}{|c|}{$F^{*}$} \\
\hline & $N=M=36$ & $N=M=44$ & $N=M=52$ \\
\hline \multicolumn{4}{|c|}{ For an impermeable wall } \\
\hline 0.1 & 1.26891 & 1.26891 & 1.26891 \\
\hline 0.2 & 1.74270 & 1.74270 & 1.74270 \\
\hline 0.3 & 2.66729 & 2.66729 & 2.66729 \\
\hline 0.4 & 4.63709 & 4.63709 & 4.63709 \\
\hline 0.5 & 9.27694 & 9.27694 & 9.27694 \\
\hline 0.6 & 21.7983 & 21.7983 & 21.7983 \\
\hline 0.7 & 63.0074 & 63.0074 & 63.0074 \\
\hline 0.8 & 245.098 & 245.098 & 245.098 \\
\hline 0.9 & 1352.57 & 1352.57 & 1352.57 \\
\hline 0.95 & 3276.05 & 3276.05 & 3276.05 \\
\hline 0.99 & 9991.09 & 9991.09 & 9991.09 \\
\hline 0.995 & 15084.2 & 15085.1 & 15085.1 \\
\hline 0.999 & 38049.3 & 37463.1 & 37369.9 \\
\hline \multicolumn{4}{|c|}{ For a wall prescribed with the far-field solute concentration profile } \\
\hline 0.1 & 1.26764 & 1.26764 & 1.26764 \\
\hline 0.2 & 1.72875 & 1.72875 & 1.72875 \\
\hline 0.3 & 2.59542 & 2.59542 & 2.59542 \\
\hline 0.4 & 4.34253 & 4.34253 & 4.34253 \\
\hline 0.5 & 8.13570 & 8.13570 & 8.13570 \\
\hline 0.6 & 17.2208 & 17.2208 & 17.2208 \\
\hline 0.7 & 42.3335 & 42.3335 & 42.3335 \\
\hline 0.8 & 127.265 & 127.264 & 127.264 \\
\hline 0.9 & 439.761 & 439.761 & 439.761 \\
\hline 0.95 & 667.882 & 667.882 & 667.882 \\
\hline 0.99 & 733.556 & 733.556 & 733.556 \\
\hline 0.995 & 733.622 & 733.626 & 733.626 \\
\hline 0.999 & 734.564 & 733.263 & 733.056 \\
\hline
\end{tabular}

solutions of the vesicle velocity converge satisfactorily (to five significant figures) for all parameters $\kappa$ and $\bar{\kappa}$ and sphere-tocylinder radius ratios $a / b$ when $\delta \leq 0.1^{\circ}$. Thus, $\delta$ was taken as $0.1^{\circ}$ in all calculations in this work.

Numerical solutions for the normalized osmophoretic force acting on a spherical vesicle on the axis of a cylindrical pore with an impermeable wall or a wall prescribed with the far-field solute concentration profile caused by an axial solute gradient, defined by Eq. 27, for the case of $\kappa=\bar{\kappa}=0$ are given in Table 1 for various values of the spacing parameter $a / b$ using the collocation technique. All of these results were obtained by choosing the number of collocation points $N(=M)$ equal to 36 , 44 , and 52 to show their convergence. The rate of convergence is rapid for small values of $a / b$ and deteriorates monotonically as the distance between the vesicle and the wall decreases. Opposite to intuition, but consistent with the reflection solution obtained by Anderson ${ }^{6}$ for an impermeable pore wall, the results in Table 1 illustrate that the osmophoretic force exerted on the vesicle increases monotonically and dramatically as the parameter $a / b$ increases. This occurs because the fluid flow accompanying the osmophoretic vesicle is opposite to the direction of its migration.

In Table 2, the collocation solutions for the osmophoretic velocity of a spherical vesicle along the axis of a cylindrical pore for different values of the parameters $\kappa, \bar{\kappa}$, and $a / b$ are presented for both cases of an impermeable wall and a wall with the imposed far-field solute concentration gradient. The velocity for the osmophoretic motion of an identical vesicle in an infinite fluid, $U_{0}$, given by Eq. 1 , is used to normalize the wall-corrected quantities. All of the results obtained under the collocation scheme converge satisfactorily to at least the significant figures shown in the table. Again, the accuracy and convergence behavior of the truncation technique is principally a function of the ratio $a / b$. For the most difficult case with $a / b=0.999$, the numbers of collocation points $M=N=52$ are sufficiently large to achieve this convergence. The dimensionless Stokes resistance coefficient $f^{*}=F^{*} /\left(U / U_{0}\right)$ as a function of $a / b$ can be calculated using the collocation solutions presented in Tables 1 and 2 for the case of $\kappa=\bar{\kappa}=0$, and the results agree well with those available in the literature. ${ }^{12,13}$

In Appendix A, an approximate analytical solution for the same osmophoretic motion as that considered here is also obtained by using a method of reflections. The vesicle velocity is given by Eq. A13, which is a power series expansion in $\lambda$ $(=a / b)$. The values of the wall-corrected normalized vesicle velocity calculated from this asymptotic solution, with the $O\left(\lambda^{8}\right)$ term neglected, are also listed in Table 2 for comparison. It can be seen that the asymptotic formula of Eq. A13 from the method of reflections for $U / U_{0}$ agrees very well with the exact results as long as $\lambda \leq 0.6$; the errors in all cases are $<10 \%$. However, the accuracy of Eq. A13 deteriorates rapidly, as expected, when the relative spacing between the vesicle and the wall becomes small. The formula of Eq. A13 always underestimates the osmophoretic velocity of the vesicle.

The collocation solutions for the normalized velocity $U / U_{0}$ of a spherical vesicle undergoing osmophoresis along the axis of a cylindrical pore as a function of the ratio $a / b$ are plotted in Figures 2 and 3 for various values of $\kappa$ and $\bar{\kappa}$. It can be seen that the boundary effect of the pore wall on osmophoretic motion is quite significant. The wall-corrected normalized mobility $U / U_{0}$ of the vesicle decreases with an increase in $\kappa$ and with a decrease in $\bar{\kappa}$ for the case of an impermeable wall (the boundary condition in Eq. 5 is used), but increases with an increase in $\kappa$ and with a decrease in $\bar{\kappa}$ for the case of a wall prescribed with the far-field solute concentration distribution (the boundary condition in Eq. 7 is used), keeping each other parameter unchanged. This decrease and increase in the vesicle mobility becomes more pronounced as $a / b$ increases. This behavior is expected, knowing that the solute concentration gradients along the vesicle surface near an impermeable wall decrease as the ratio $\kappa /(1+\bar{\kappa})$ increases and these concentration gradients near a wall with the imposed far-field concentration distribution increase as $\kappa /(1+\bar{\kappa})$ increases (see the analysis in Appendix A). When $\kappa=1+\bar{\kappa}$, the two types of pore wall will result in the same effects on the osmophoretic motion of the vesicle. In this particular case, the effect of solutal interaction between the vesicle and the wall disappears, and the relative osmophoretic mobility of the vesicle is independent of the value of either $\kappa$ or $\bar{\kappa}$ and increases monotonically with $a / b$, attributed solely to the hydrodynamic enhancement exerted by the pore wall.

Examination of the results shown in Table 2 and Figure 3 reveals an interesting feature. For the case of an impermeable pore wall under the situation of $\kappa \gg 1+\bar{\kappa}$, the osmophoretic mobility of the vesicle increases with an increase in $a / b$ if $a / b$ is small, but decreases from a maximum with increasing $a / b$ if $a / b$ is sufficiently large. This feature that $U / U_{0}$ may not be a monotonic function of $a / b$ is understandable because the wall 
Table 2. Normalized Osmophoretic Velocity of a Spherical Vesicle along the Axis of a Circular Cylindrical Pore Computed from the Exact Boundary-Collocation Solution and the Asymptotic Method-of-Reflection Solution

\begin{tabular}{|c|c|c|c|c|c|c|}
\hline \multirow[b]{3}{*}{$a / b$} & \multicolumn{6}{|c|}{$U / U_{0}$} \\
\hline & \multicolumn{2}{|c|}{$\kappa=\bar{\kappa}=0$} & \multicolumn{2}{|c|}{$\kappa=10, \bar{\kappa}=0$} & \multicolumn{2}{|c|}{$\kappa=0, \bar{\kappa}=10$} \\
\hline & Exact Solution & Asymptotic Solution & Exact Solution & Asymptotic Solution & Exact Solution & Asymptotic Solution \\
\hline \multicolumn{7}{|c|}{ For an impermeable wall } \\
\hline 0.1 & 1.00494 & 1.00494 & 1.00294 & 1.00294 & 1.00494 & 1.00494 \\
\hline 0.2 & 1.03881 & 1.03880 & 1.02241 & 1.02238 & 1.03881 & 1.03880 \\
\hline 0.3 & 1.12807 & 1.12787 & 1.06923 & 1.06860 & 1.12809 & 1.12787 \\
\hline 0.4 & 1.29737 & 1.29548 & 1.14322 & 1.13718 & 1.29762 & 1.29548 \\
\hline 0.5 & 1.57533 & 1.56464 & 1.23151 & 1.19813 & 1.57673 & 1.56464 \\
\hline 0.6 & 2.00894 & 1.96342 & 1.31266 & 1.18229 & 2.01496 & 1.96342 \\
\hline 0.7 & 2.70070 & 2.53329 & 1.36435 & 0.96529 & 2.72339 & 2.53329 \\
\hline 0.8 & 3.93737 & 3.34016 & 1.36982 & 0.34891 & 4.02282 & 3.34016 \\
\hline 0.9 & 6.94877 & 4.48847 & 1.31876 & -0.96016 & 7.35950 & 4.48847 \\
\hline 0.95 & 11.5622 & & 1.26908 & & 12.8505 & \\
\hline 0.99 & 33.1003 & & 1.21558 & & 41.8625 & \\
\hline 0.995 & 50.1532 & & 1.20791 & & 66.9547 & \\
\hline 0.999 & 124 & & 1.20 & & 186 & \\
\hline \multicolumn{7}{|c|}{ For a wall prescribed with the far-field solute concentration profile } \\
\hline 0.1 & 1.00393 & 1.00393 & 1.00445 & 1.00445 & 1.00393 & 1.00393 \\
\hline 0.2 & 1.03049 & 1.03047 & 1.03475 & 1.03472 & 1.03049 & 1.03047 \\
\hline 0.3 & 1.09767 & 1.09725 & 1.11309 & 1.11267 & 1.09766 & 1.09725 \\
\hline 0.4 & 1.21495 & 1.21139 & 1.25609 & 1.25292 & 1.21482 & 1.21139 \\
\hline 0.5 & 1.38154 & 1.36332 & 1.47567 & 1.46041 & 1.38086 & 1.36332 \\
\hline 0.6 & 1.58707 & 1.51999 & 1.78290 & 1.72894 & 1.58447 & 1.51999 \\
\hline 0.7 & 1.81456 & 1.61732 & 2.19588 & 2.04041 & 1.80643 & 1.61732 \\
\hline 0.8 & 2.04444 & 1.55219 & 2.75628 & 2.36504 & 2.02232 & 1.55219 \\
\hline 0.9 & 2.25924 & 1.17355 & 3.58040 & 2.66238 & 2.20485 & 1.17355 \\
\hline 0.95 & 2.35716 & & 4.19822 & & 2.27442 & \\
\hline 0.99 & 2.43025 & & 4.92137 & & 2.31569 & \\
\hline 0.995 & 2.43906 & & 5.03852 & & 2.31977 & \\
\hline 0.999 & 2.446 & & 5.141 & & 2.323 & \\
\hline
\end{tabular}

effect of hydrodynamic enhancement on the vesicle is in the competition with the wall effect of solutal retardation when a vesicle with a large value of $\kappa /(1+\bar{\kappa})$ is undergoing osmo-

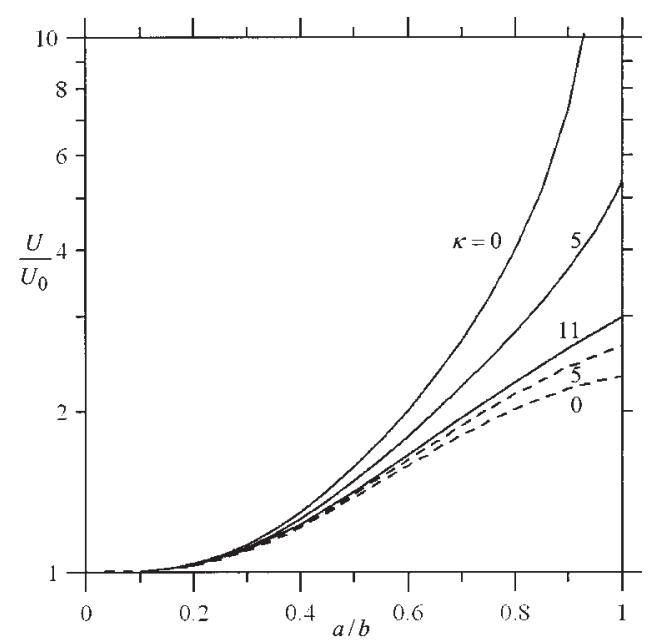

Figure 2. Plots of the normalized osmophoretic velocity of a spherical vesicle with $\bar{\kappa}=10$ along the axis of a circular cylindrical pore vs. the ratio $a / b$ for various values of $\boldsymbol{\kappa}$.

The solid curves represent the case of an impermeable wall, and the dashed curves denote the case of a wall on which the far-field solute concentration profile is imposed. phoretic motion near an impermeable wall. Under the situations of a small to moderate value of $\kappa /(1+\bar{\kappa})$, the osmophoretic mobility of the vesicle near the impermeable wall is a

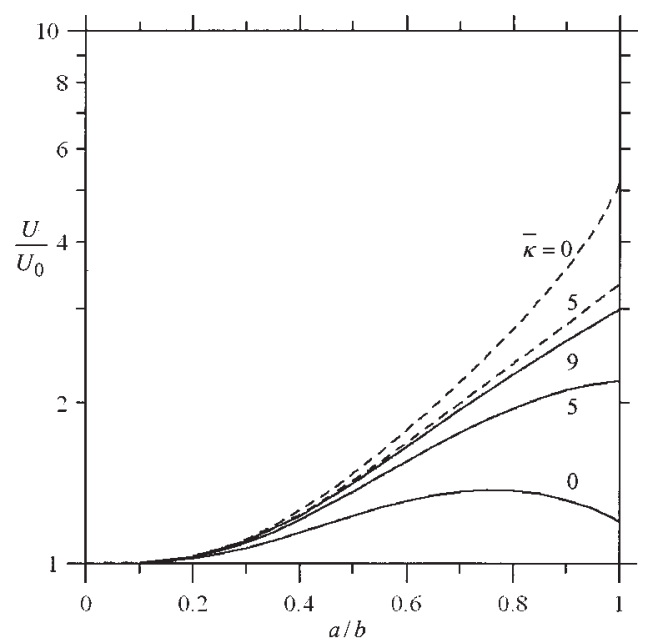

Figure 3. Plots of the normalized osmophoretic velocity of a spherical vesicle with $\kappa=10$ along the axis of a circular cylindrical pore vs. the ratio $a / b$ for various values of $\bar{\kappa}$.

The solid curves represent the case of an impermeable wall, and the dashed curves denote the case of a wall on which the far-field solute concentration profile is imposed. 


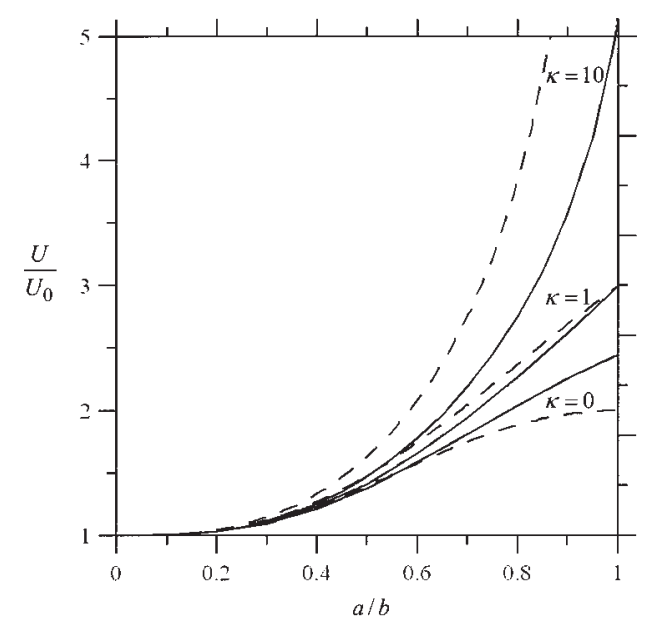

Figure 4. A comparison of the normalized osmophoretic velocities of a spherical vesicle with $\bar{\kappa}=0$ along the axis of a circular cylindrical pore (solid curves) and located at the center of a spherical pore (dashed curves) for various values of $a / b$ and $\kappa$ (or $G$ ).

The solute concentration at the pore wall is taken as the distribution giving rise to the constant gradient $E_{\infty}$ in the absence of the vesicle.

monotonic increasing function of $a / b$. For any case that a linear concentration profile is prescribed on the pore wall, which is consistent with the far-field solute distribution, the osmophoretic mobility of the vesicle is also a monotonic increasing function of $a / b$.

For the osmophoretic motion of a spherical vesicle of radius $a$ situated at the center of a spherical cavity of radius $b$, the normalized vesicle mobility can be determined analytically as

$$
\frac{U}{U_{0}}=\frac{1+5 \lambda^{3}-6 \lambda^{5}}{\left(1+G \lambda^{3}\right)\left(1-\lambda^{5}\right)}
$$

where

$$
G=\frac{1+\bar{\kappa}-\kappa}{2+2 \bar{\kappa}+\kappa}
$$

and $\lambda=a / b$. Obviously, $-1 \leq G \leq 1 / 2$, with the upper and lower bounds occurring at the limits $\kappa \ll 1+\bar{\kappa}$ and $\kappa \gg 2$ (1 $+\bar{\kappa}$ ), respectively. To obtain Eq. 28, the solute concentration at the cavity wall is taken as the distribution giving rise to the constant gradient $E_{\infty}$ in the cavity when the vesicle does not exist. This normalized osmophoretic mobility, which depends on one parameter $G$ composed of $\kappa$ and $\bar{\kappa}$, increases monotonically from unity at $\lambda=0$ to $3 /(1+G)$ as $\lambda \rightarrow 1$. A comparison of the collocation results for the osmophoretic mobility of the vesicle in a corresponding cylindrical pore with Eq. 28 is presented in Figure 4. It can be seen that the boundary effects on osmophoresis in cylindrical and spherical pores are quite similar and the mobility enhancement in the cylindrical pore can be greater or smaller than that in the spherical cavity with the same values of $\kappa, \bar{\kappa}$, and $a / b$. The agreement between the numerical values of the two wall-corrected mobilities for the case of $G=0$ (the osmophoretic mobility of the vesicle along the axis of the cylindrical pore becomes independent of the absolute values of $\kappa$ and $\bar{\kappa}$ in this case) is quite good, with the difference being within $5.5 \%$. The similarity in the boundary effects in these pore geometries reflects the fact that the deformation of solute flux field and the viscous enhancement in the cylindrical pore are greatest at the point of closest approach of the vesicle to the pore wall, with the geometry of this region being analogous to that of a vesicle in the spherical cavity. Note that the osmophoresis of a vesicle in a spherical cavity might represent a real system of the chemotactic movement of a vesicle in a biological cell.

\section{Concluding Remarks}

The exact numerical solution and approximate analytical solution for the quasi-steady osmophoretic motion of a spherical vesicle with a thin, rigid membrane along the axis of a cylindrical pore have been obtained in this work by using the boundary-collocation technique and the method of reflections, respectively. Both the cases of an impermeable wall and of a wall with the imposed far-field solute concentration distribution were examined in the limit of vanishingly small Reynolds and Peclet numbers. It has been found that the agreement between the collocation solution and the reflection solution is quite good, and the boundary effect on osmophoretic motion of a vesicle is significant and complicated. The osmophoretic mobility of a vesicle in the pore is generally, but not necessarily, a monotonic increasing function of the separation parameter $a / b$. For the case of an impermeable pore wall with $\kappa \gg$ $1+\bar{\kappa}$, the vesicle mobility may increase with an increase in $a / b$ if $a / b$ is small, and then decrease with an increase in $a / b$ if $a / b$ becomes large. This behavior reflects the competition between the hydrodynamic enhancement exerted by the pore wall on the vesicle migration and the possible osmotic retardation arising from the solutal interaction between the vesicle and the wall.

The osmophoretic mobility of a spherical vesicle on the median plane between two parallel plane walls was calculated in a previous work ${ }^{9}$ for various values of the parameters $\kappa, \bar{\kappa}$, and $a / b$, where $b$ is the distance between the center of the vesicle and each of the plane walls. It was also found that, for the case of impermeable walls under the situation of $\kappa \gg 1+$ $\bar{\kappa}$, the vesicle mobility first increases and then decreases with increasing $a / b$. When the gaps between the vesicle and the plane walls turn thin, however, the vesicle can even migrate slower than it would if $a / b=0$ (by as much as $22 \%$ for an example of $\kappa=10, \bar{\kappa}=0$, and $a / b=0.999$ ). Exactly the same tendency of the dependency of $U / U_{0}$ on $a / b$ can be observed for the case in which the two plane walls are prescribed with the far-field solute concentration profile under the situation of $\kappa \ll$ $1+\bar{\kappa}$. This difference between the boundary effects on osmophoresis in a circular cylindrical pore and in a slit pore is striking, suggesting that the effect of viscous interactions is stronger or the effect of solutal interactions is weaker in a circular pore than in a slit pore. In general, and quite surprisingly, the net boundary effect on osmophoresis of a vesicle is very much stronger in a circular pore than in a slit.

The membranes of different types of vesicles can vary in physicochemical properties such as the deformability and strength. In this paper, the membrane of the vesicle has been assumed rigid during the osmophoretic motion, and the possi- 
ble effects of local and total membrane deformations as well as the development of stress gradients in the membrane were not considered. Zinemanas and $\mathrm{Nir}^{14}$ theoretically examined the unsteady osmophoresis of a deformable vesicle in an unbounded fluid accounting for the temporal changes in the volume and concentration of the encapsulated solution resulting from vesicle shrinking and swelling (occurring when the vesicle is placed in high and low osmotic pressure environments, respectively). It was found that the osmophoresis of deformable vesicles has a transient dynamics and is ultimately halted, given that the surface tractions eventually balance the osmotic load.

\section{Acknowledgments}

This research was partly supported by the National Science Council of the Republic of China.

\section{Notation}

$a=$ radius of the vesicle, $\mathrm{m}$

$A=$ osmophoretic mobility defined by Eq. $1 \mathrm{~b}, \mathrm{~m}^{5} \mathrm{~s}^{-1}$

$b=$ radius of the pore, $\mathrm{m}$

$B_{\mathrm{n}}, D_{\mathrm{n}}=$ coefficients in Eq. 22 or Eq. 23 for the flow field, $\mathrm{m}^{n+2} \mathrm{~s}^{-1}$, $\mathrm{m}^{n} \mathrm{~s}^{-1}$

$C=$ solute concentration field outside the vesicle, $\mathrm{m}^{-3}$

$C_{0}=$ value of $C_{\infty}$ at the position of vesicle center, $\mathrm{m}^{-3}$

$C_{1}=$ solute concentration field inside the vesicle, $\mathrm{m}^{-3}$

$\vec{C}=$ average solute concentration inside the vesicle, $\mathrm{m}^{-3}$

$C_{\infty}=$ prescribed solute concentration field defined by Eq. $6, \mathrm{~m}^{-3}$

$\mathbf{e}_{r}, \mathbf{e}_{z}=$ unit vectors in $r$ and $z$ directions

$E_{\infty}=\left|\nabla C_{\infty}\right|, \mathrm{m}^{-4}$

$F^{*}=$ normalized osmophoretic force on the vesicle defined by Eq. 27

$G=$ dimensionless parameter defined by Eq. 29

$G_{n}^{-1 / 2}=$ Gegenbauer polynomial of the first kind of order $n$ and degree $-1 / 2$

$I_{\mathrm{n}, K \mathrm{n}}=$ modified Bessel functions of the first and second kinds

$L_{\mathrm{p}}=$ hydraulic coefficient of the vesicle membrane, $\mathrm{m}^{2} \mathrm{~s} \mathrm{~kg}^{-1}$

$P_{\mathrm{m}}=$ Legendre polynomial of order $m$

$r=$ radial spherical coordinate, $\mathrm{m}$

$R=$ the gas constant, $\mathrm{J} \mathrm{K}^{-1]}$

$T=$ absolute temperature, $\mathrm{K}$

$T_{\mathrm{m}}, \bar{T}_{m}=$ coefficients in Eqs. $10-12$ for the solute concentration field, $\mathrm{m}^{m+2}, \mathrm{~m}^{-m+1}$

$\mathbf{U}, U=$ osmophoretic velocity of the vesicle, $\mathrm{m} \mathrm{s}^{-1}$

$\mathbf{U}_{0}, U 0=$ osmophoretic velocity of an isolated vesicle defined by Eq. $1, \mathrm{~m} \mathrm{~s}^{-1}$

$\mathbf{v}=$ velocity field of the fluid, $\mathrm{m} \mathrm{s}^{-1}$

$z=$ axial cylindrical coordinate, $\mathrm{m}$

\section{Greek letters}

$\gamma_{n}^{(i)}=$ functions of $r$ and $\theta$ defined by Eqs. B3-B6, $\mathrm{m}^{-n-1}$ or $\mathrm{m}^{-n+1}$

$\delta_{m}^{(1)}, \delta_{m}^{(2)}=$ functions of $r$ and $\theta$ defined by Eqs. B1 and B2, $\mathrm{m}^{-m-1}$, $\mathrm{m}^{-m-2}$

$\eta=$ viscosity of the fluid, $\mathrm{kg} \mathrm{m}^{-1} \mathrm{~s}^{-1}$

$\theta, \phi=$ angular spherical coordinates

$\kappa, \bar{\kappa}=$ dimensionless parameters defined by Eq. 2

$\lambda=a / b$

$\rho=$ radial cylindrical coordinate, $\mathrm{m}$

$\Psi=$ Stokes stream function for the fluid flow, $\mathrm{m}^{3} \mathrm{~s}^{-1}$

\section{Subscripts}

$\mathrm{p}=$ vesicle

$\mathrm{w}=$ wall

\section{Literature Cited}

1. Gordon LGM. Osmophoresis. J Phys Chem. 1981;85:1753-1755

2. Pope CG. Investigation of osmophoresis. J Phys Chem. 1982;86:18691870.

3. Anderson JL. Movement of a semipermeable vesicle through an osmotic gradient. Phys Fluids. 1983;26:2871-2879.

4. Anderson JL. Shape and permeability effects on osmophoresis. Physicochem Hydrodyn. 1984;5:205-216.

5. Nardi J, Bruinsma R, Sackmann E. Vesicles as osmotic motors. Phys Rev Lett. 1999;82:5168-5171.

6. Anderson JL. Transport mechanisms of biological colloids. Ann NY Acad Sci (Biochem Eng IV). 1986;469:166-177.

7. Berg HC, Turner L. Chemotaxis of bacteria in glass capillary arrays. Biophys J. 1990;58:919-930.

8. Keh HJ, Yang FR. Boundary effects on osmophoresis: Motion of a vesicle in an arbitrary direction with respect to a plane wall. Chem Eng Sci. 1993;48:3555-3563.

9. Chen PY, Keh HJ. Boundary effects on osmophoresis: Motion of a spherical vesicle parallel to two plane walls. Chem Eng Sci. 2003;58: 4449-4464.

10. Keh HJ, Chiou JY. Electrophoresis of a colloidal sphere in a circular cylindrical pore. AIChE J. 1996;42:1397-1406.

11. O'Brien V. Form factors for deformed spheroids in stokes flow. AIChE J. 1968; 14:870-875.

12. Leichtberg S, Pfeffer R, Weinbaum S. Stokes flow past finite coaxial clusters of spheres in a circular cylinder. Int J Multiphase Flow. 1976;3:147-169.

13. Happel J, Brenner H. Low Reynolds Number Hydrodynamics. Dordrecht, The Netherlands: Nijhoff; 1983.

14. Zinemanas D, Nir A. Osmophoretic motion of deformable particles. Int J Multiphase Flow. 1995;21:787-800.

15. Keh HJ, Tu HJ. Osmophoresis in a dilute suspension of spherical vesicles. Int J Multiphase Flow. 2000;26:125-145.

\section{Appendix A: Analysis of the Osmophoresis of a Spherical Vesicle in a Circular Cylindrical Pore by a Method of Reflections}

In this appendix, we analyze the quasi-steady osmophoretic motion of an arbitrary spherical vesicle with a thin, rigid membrane along the axis of a long circular cylindrical pore, as shown in Figure 1, by a method of reflections. The effect of the pore wall on the osmophoretic velocity $\mathbf{U}$ of the vesicle is sought in an expansion of $\lambda$, which equals $a / b$, the ratio of vesicle-to-pore radii.

For the osmophoresis of a vesicle in a pore with an impermeable wall, the governing Eqs. 3a and 14 must be solved by satisfying the boundary conditions 4-6 and 17-19. The method-of-reflection solution consists of the following series, whose terms depend on increasing powers of $\lambda$

$$
\begin{gathered}
C=C_{0}-E_{\infty} z+C_{\mathrm{p}}^{(1)}+C_{\mathrm{w}}^{(1)}+C_{\mathrm{p}}^{(2)}+C_{\mathrm{w}}^{(2)}+\cdots \\
\mathbf{v}=\mathbf{v}_{\mathrm{p}}^{(1)}+\mathbf{v}_{\mathrm{w}}^{(1)}+\mathbf{v}_{\mathrm{p}}^{(2)}+\mathbf{v}_{\mathrm{w}}^{(2)}+\cdots
\end{gathered}
$$

where subscripts $\mathrm{w}$ and $\mathrm{p}$ represent the reflections from wall and vesicle, respectively, and the superscript ( $i$ ) denotes the $i$ th reflection from that surface. In these series, all the expansion sets of the solute concentration and fluid velocity for the solution phase outside the vesicle must satisfy Eqs. 3a and 14 . The advantage of this method is that it is necessary to consider boundary conditions associated with only one surface at a time.

According to the system of Eq. A1, the velocity of the vesicle can also be expressed in a series form as 


$$
\mathbf{U}=U_{0} \mathbf{e}_{z}+\mathbf{U}^{(1)}+\mathbf{U}^{(2)}+\cdots
$$

In this expression, $U_{0}=A E_{\infty}$ is the osmophoretic velocity of an identical vesicle suspended freely in the unbounded solution phase given by Eq. $1 ; \mathbf{U}^{(i)}$ is related to $\nabla C_{\mathrm{w}}^{(i)}$ and $\mathbf{v}_{\mathrm{w}}^{(i)}$ by ${ }^{15}$

$$
\mathbf{U}^{(i)}=-A\left[\nabla C_{\mathrm{w}}^{(i)}\right]_{0}+\left[\mathbf{v}_{\mathrm{w}}^{(i)}\right]_{0}+\frac{a^{2}}{6}\left[\nabla^{2} \mathbf{v}_{\mathrm{w}}^{(i)}\right]_{0}
$$

where the subscript 0 to variables inside brackets denotes evaluation at the position of the vesicle center.

The solution for the first reflected fields from the vesicle is

$$
\begin{gathered}
C_{\mathrm{p}}^{(1)}=-G E_{\infty} a^{3} r^{-2} \cos \theta \\
\mathbf{v}_{\mathrm{p}}^{(1)}=-U_{0} a^{3} r^{-3}\left(2 \cos \theta \mathbf{e}_{r}+\sin \theta \mathbf{e}_{\theta}\right)
\end{gathered}
$$

where $G$ was defined by Eq. 29. The velocity distribution shown in Eq. A4b is identical to the irrotational flow surrounding a rigid sphere moving with velocity $-2 U_{0} \mathbf{e}_{z}$.

The boundary conditions for the $i$ th reflected fields from the wall are derived from Eqs. 5, 6, 18, and 19, as follows

$$
\begin{gathered}
\rho=b: \frac{\partial C_{\mathrm{w}}^{(i)}}{\partial \rho}=-\frac{\partial C_{\mathrm{p}}^{(i)}}{\partial \rho} \\
\mathbf{v}_{\mathrm{w}}^{(i)}=-\mathbf{v}_{\mathrm{p}}^{(i)} \\
|z| \rightarrow \infty: \quad C_{\mathrm{w}}^{(i)} \rightarrow 0 \\
\mathbf{v}_{\mathrm{w}}^{(i)} \rightarrow \mathbf{0}
\end{gathered}
$$

The solution of $C_{\mathrm{w}}^{(1)}$ is obtained by applying Fourier sine transforms on the variable $z$ in Eqs. $3 a$ and A5a and A5c (taking $i=1$ ), with the result

$$
C_{\mathrm{w}}^{(1)}=-\frac{2}{\pi} G E_{\infty} a \lambda^{2} \int_{0}^{\infty} \omega \frac{K_{1}(\omega)}{I_{1}(\omega)} I_{0}\left(\frac{\rho}{b} \omega\right) \sin \left(\frac{z}{b} \omega\right) d \omega
$$

where $I_{n}(\omega)$ and $K_{n}(\omega)$ are modified Bessel functions of the first kind and second kind, respectively, of order $n . \mathbf{v}_{\mathrm{w}}^{(1)}$ can be solved by applying Fourier cosine transforms twice to the Stokes Eq. 14 and boundary conditions A5b and A5d, which results in

$$
\begin{aligned}
\mathbf{v}_{\mathrm{w}}^{(1)}=- & \frac{2}{\pi} U_{0} \lambda^{3} \int_{0}^{\infty}\left\{\left[\alpha(\omega) \frac{\rho}{b} \omega I_{0}\left(\frac{\rho}{b} \omega\right)\right.\right. \\
& \left.-\beta(\omega) I_{1}\left(\frac{\rho}{b} \omega\right)\right] \sin \left(\frac{z}{b} \omega\right) \mathbf{e}_{\rho}+\left[2 \alpha(\omega) I_{0}\left(\frac{\rho}{b} \omega\right)\right. \\
+ & \left.\left.\alpha(\omega) \frac{\rho}{b} \omega I_{1}\left(\frac{\rho}{b} \omega\right)-\beta(\omega) I_{0}\left(\frac{\rho}{b} \omega\right)\right] \cos \left(\frac{z}{b} \omega\right) \mathbf{e}_{z}\right\} d \omega
\end{aligned}
$$

where

$$
\begin{gathered}
\alpha(\omega)=\left\{\left[I_{1}(\omega)\right]^{2}-I_{0}(\omega) I_{2}(\omega)\right\}^{-1} \\
\beta(\omega)=\omega^{2}\left[I_{1}(\omega) K_{1}(\omega)+I_{0}(\omega) K_{2}(\omega)\right] \alpha(\omega)
\end{gathered}
$$

The contributions of $C_{\mathrm{w}}^{(1)}$ and $\mathbf{v}_{\mathrm{w}}^{(1)}$ to the vesicle velocity are determined by using Eq. A3

$$
\begin{gathered}
\mathbf{U}_{\mathrm{s}}^{(1)}=-A\left[\nabla C_{\mathrm{w}}^{(1)}\right]_{r=0}=d_{1} G \lambda^{3} U_{0} \mathbf{e}_{z} \\
\mathbf{U}_{\mathrm{h}}^{(1)}=\left[\mathbf{v}_{\mathrm{w}}^{(1)}+\frac{a^{2}}{6} \nabla^{2} \mathbf{v}_{\mathrm{w}}^{(1)}\right]_{r=0}=\left(d_{2} \lambda^{3}-d_{3} \lambda^{5}\right) U_{0} \mathbf{e}_{z} \\
\mathbf{U}^{(1)}=\mathbf{U}_{\mathrm{s}}^{(1)}+\mathbf{U}_{\mathrm{h}}^{(1)}=\left[\left(d_{2}+d_{1} G\right) \lambda^{3}-d_{3} \lambda^{5}\right] U_{0} \mathbf{e}_{z}
\end{gathered}
$$

where

$$
\begin{gathered}
d_{1}=\frac{2}{\pi} \int_{0}^{\infty} \omega^{2} \frac{K_{1}(\omega)}{I_{1}(\omega)} d \omega=1.59365 \\
d_{2}=\frac{2}{\pi} \int_{0}^{\infty}[\beta(\omega)-2 \alpha(\omega)] d \omega=4.17338 \\
d_{3}=\frac{2}{3 \pi} \int_{0}^{\infty} \omega^{2} \alpha(\omega) d \omega=3.79264
\end{gathered}
$$

Equation A8a shows that the reflected solute concentration field from the impermeable wall can increase (if $G>0$ or $\kappa<$ $1+\bar{\kappa}$ ) or decrease (if $G<0$ or $\kappa>1+\bar{\kappa}$ ) the osmophoretic velocity of the vesicle, whereas Eq. A8b indicates that the reflected velocity field is to increase this velocity; the net effect of the reflected fields is expressed by Eq. A8c, which can enhance or retard the movement of the vesicle, depending on the combination of the values of $G$ (or $\kappa$ and $\bar{\kappa}$ ) and $\lambda$. When $G=0$ (or $\kappa=1+\bar{\kappa}$ ), the reflected concentration field makes no contribution to the osmophoretic velocity. Equation A8c indicates that the wall correction to the vesicle velocity is $O\left(\lambda^{3}\right)$, which is weaker than that obtained for the corresponding sedimentation problem, in which the leading boundary effect is $O(\lambda)$. Note that the necessary condition for the wall retardation on the osmophoretic motion to occur is $\kappa \gg 1+$ $\bar{\kappa}$ and a value of $\lambda$ close to unity such that the relation $d_{3} \lambda^{5}>$ $\left(d_{2}+d_{1} G\right) \lambda^{3}$ is warranted.

The solution for the second reflected fields from the vesicle is

$$
\begin{gathered}
C_{\mathrm{p}}^{(2)}=-E_{\infty}\left[d_{1} G^{2} \lambda^{3} a^{3} r^{-2} \cos \theta+O\left(\lambda^{5} a^{5}\right)\right] \\
\mathbf{v}_{\mathrm{p}}^{(2)}=-U_{0}\left[d_{1} G \lambda^{3} a^{3} r^{-3}\left(2 \cos \theta \mathbf{e}_{r}+\sin \theta \mathbf{e}_{\theta}\right)+O\left(\lambda^{5} a^{3}\right)\right]
\end{gathered}
$$

The boundary conditions for the second reflected fields from the wall are obtained by substituting the results of $C_{\mathrm{p}}^{(2)}$ and $\mathbf{v}_{\mathrm{p}}^{(2)}$ 
into Eq. A5, with which Eqs. 3a and 14 can be solved as before to yield

$$
\begin{gathered}
C_{\mathrm{w}}^{(2)}=d_{1}^{2} G^{2} \lambda^{6} E_{\infty} z+O\left(\lambda^{7}\right) \\
\mathbf{v}_{\mathrm{w}}^{(2)}=d_{1} d_{2} G \lambda^{6} U_{0} \mathbf{e}_{z}+O\left(\lambda^{7}\right)
\end{gathered}
$$

The contribution of the second reflected fields to the velocity of the vesicle is obtained by combining Eqs. A3 and A11, which gives

$$
\mathbf{U}^{(2)}=\left[\left(d_{1} d_{2} G+d_{1}^{2} G^{2}\right) \lambda^{6}+O\left(\lambda^{8}\right)\right] U_{0} \mathbf{e}_{z}
$$

The error for $\mathbf{U}^{(2)}$ is $O\left(\lambda^{8}\right)$ because the $O\left(\lambda^{7}\right)$ terms in the expansions of $\nabla C_{\mathrm{w}}^{(2)}$ and $\mathbf{v}_{\mathrm{w}}^{(2)}$ vanish at the position of the vesicle center.

Obviously, $\mathbf{U}^{(3)}$ will be $O\left(\lambda^{9}\right)$. With the substitution of Eqs. $\mathrm{A} 8 \mathrm{c}$ and $\mathrm{A} 12$ into Eq. A2, the vesicle velocity can be expressed as $\mathbf{U}=U \mathbf{e}_{z}$ with

$$
\begin{array}{r}
U=U_{0}\left[1+\left(d_{2}+d_{1} G\right) \lambda^{3}-d_{3} \lambda^{5}+\left(d_{1} d_{2} G+d_{1}^{2} G^{2}\right) \lambda^{6}\right. \\
\left.+O\left(\lambda^{8}\right)\right]
\end{array}
$$

For the special case of $\kappa=\bar{\kappa}=0$ or $G=1 / 2$, Eq. A13 is exactly the same as the result obtained by Anderson. ${ }^{6}$ Note that the normalized osmophoretic mobility $U / U_{0}$, correct to $O\left(\lambda^{6}\right)$, depends on one parameter $G$ composed of $\kappa$ and $\bar{\kappa}$.

For the case that a linear solute concentration profile is prescribed on the pore wall, which is consistent with the far-field solute distribution (that is, the boundary condition 5 is replaced by Eq. 7), the series expansions $\mathrm{A} 1$ and $\mathrm{A} 2$, the solution of $C_{\mathrm{p}}^{(1)}$ and $\mathbf{v}_{\mathrm{p}}^{(1)}$ in Eq. A4, and the boundary conditions for $C_{\mathrm{w}}^{(\mathrm{i})}$ and $\mathbf{v}_{\mathrm{w}}^{(\mathrm{i})}$ in Eqs. A5b-A5d are still valid, although Eq. A5a becomes

$$
\rho=b: \quad C_{\mathrm{w}}^{(i)}=-C_{\mathrm{p}}^{(i)}
$$

The solution of $C_{\mathrm{w}}^{(1)}$, satisfying Eqs. 3a, A14, and A5c, is

$$
C_{\mathrm{w}}^{(1)}=\frac{2}{\pi} G E_{\infty} a \lambda^{2} \int_{0}^{\infty} \omega \frac{K_{0}(\omega)}{I_{0}(\omega)} I_{0}\left(\frac{\rho}{b} \omega\right) \sin \left(\frac{z}{b} \omega\right) d \omega
$$

whereas the solution of $\mathbf{v}_{\mathrm{w}}^{(1)}$ is unchanged from Eq. A6b. The results of the following reflected fields and of the vesicle velocity are also obtained from Eqs. A $8-\mathrm{A} 13$ by replacing $d_{1}$ by $-\bar{d}_{1}$ and $d_{1}^{2}$ by $-\bar{d}_{1}^{2}$, where

$$
\bar{d}_{1}=\frac{2}{\pi} \int_{0}^{\infty} \omega^{2} \frac{K_{0}(\omega)}{I_{0}(\omega)} d \omega=0.411822
$$

Thus, contrary to the effect of an impermeable pore wall, the reflected concentration field from a wall with the imposed far-field concentration distribution reduces the osmophoretic velocity of the vesicle if $G>0$ or $\kappa<1+\bar{\kappa}$ and enhances this velocity if $G<0$ or $\kappa>1+\bar{\kappa}$. When $G=0$ or $\kappa=1+\bar{\kappa}$, the two types of pore wall will produce the same effects (with no contribution from the reflected solute concentration field) on the osmophoretic motion of the vesicle and the normalized vesicle velocity becomes independent of the absolute values of $\kappa$ and $\bar{\kappa}$. Correct to $O\left(\lambda^{5}\right)$, the net effect of the pore wall prescribed with the far-field solute concentration distribution always enhances the osmophoretic migration of a vesicle.

\section{Appendix B: Definitions of Some Functions in the Analysis Section}

The functions $\delta_{m}^{(1)}$ and $\delta_{m}^{(2)}$ in Eqs. 12 and 13 are defined by

$$
\begin{gathered}
\delta_{m}^{(1)}(r, \theta) \\
\begin{array}{c}
\frac{2(-1)^{(m-2 v+1) / 2}}{\pi m !} \int_{0}^{\infty} \omega^{m} \frac{K_{v}(b \omega)}{I_{v}(b \omega)} I_{0}(\omega r \sin \theta) \sin (\omega r \cos \theta) d \omega \\
+r^{-m-1} P_{m}(\cos \theta) \quad(\mathrm{B} 1) \\
\delta_{m}^{(2)}(r, \theta)=\frac{2(-1)^{(m-2 v+1) / 2}}{\pi m !} \int_{0}^{\infty} \omega^{m+1} \frac{K_{v}(b \omega)}{I_{v}(b \omega)} \\
{\left[I_{1}(\omega r \sin \theta) \sin (\omega r \cos \theta) \sin \theta+I_{0}(\omega r \sin \theta)\right.}
\end{array}
\end{gathered}
$$$$
\times \cos (\omega r \cos \theta) \cos \theta] d \omega-(m+1) r^{-m-2} P_{m}(\cos \theta)
$$

where $I_{\nu}$ and $K_{v}$ are the modified Bessel functions of the first and second kinds, respectively, of order $\nu ; \nu=1$ if Eq. 5 is used for the boundary condition of the solute concentration field at the pore wall and $\nu=0$ if Eq. 7 is used.

The functions $\gamma_{n}^{(i)}$ for $i=1,2,3$, and 4 in Eqs. 23 and 24 are defined, respectively, by

$$
\begin{aligned}
\gamma_{n}^{(1)}(r, \theta)= & \int_{0}^{\infty}\left[S_{1}(\omega) I_{0}(\omega r \sin \theta) r \sin \theta\right. \\
+ & \left.S_{3}(\omega) I_{1}(\omega r \sin \theta)\right] \omega \sin (\omega r \cos \theta) d \omega \\
& -r^{-n-1}(n+1) G_{n+1}^{-1 / 2}(\cos \theta) \csc \theta
\end{aligned}
$$

$$
\begin{aligned}
\gamma_{n}^{(2)}(r, \theta) & =\int_{0}^{\infty}\left[S_{2}(\omega) I_{0}(\omega r \sin \theta) r \sin \theta\right. \\
& \left.+S_{4}(\omega) I_{1}(\omega r \sin \theta)\right] \omega \sin (\omega r \cos \theta) d \omega-r^{-n+1}[(n \\
& \left.+1) G_{n+1}^{-1 / 2}(\cos \theta) \csc \theta-2 G_{n}^{-1 / 2}(\cos \theta) \cot \theta\right]
\end{aligned}
$$

$$
\begin{aligned}
& \gamma_{n}^{(3)}(r, \theta)=\int_{0}^{\infty}\left\{S_{1}(\omega)\left[2 I_{0}(\omega r \sin \theta)+I_{1}(\omega r \sin \theta) \omega r \sin \theta\right]\right. \\
& \left.\quad+\omega S_{3}(\omega) I_{0}(\omega r \sin \theta)\right\} \times \cos (\omega r \cos \theta) d \omega-r^{-n-1} P_{n}(\cos \theta)
\end{aligned}
$$

$\gamma_{n}^{(4)}(r, \theta)=\int_{0}^{\infty}\left\{S_{2}(\omega)\left[2 I_{0}(\omega r \sin \theta)+I_{1}(\omega r \sin \theta) \omega r \sin \theta\right]\right.$ 
$\left.+\omega S_{4}(\omega) I_{0}(\omega r \sin \theta)\right\} \cos (\omega r \cos \theta) d \omega$

$$
-r^{-n+1}\left[2 G_{n}^{-1 / 2}(\cos \theta)+P_{n}(\cos \theta)\right]
$$

In Eqs. B3-B6,

$$
S_{1}(\omega)=\frac{A_{n}^{(1)}(\omega) I_{0}(b \omega)-A_{n}^{(3)}(\omega) I_{1}(b \omega)}{b \omega I_{0}^{2}(b \omega)-2 I_{0}(b \omega) I_{1}(b \omega)-b \omega I_{1}^{2}(b \omega)}
$$

$$
S_{2}(\omega)=\frac{A_{n}^{(2)}(\omega) I_{0}(b \omega)-A_{n}^{(4)}(\omega) I_{1}(b \omega)}{b \omega I_{0}^{2}(b \omega)-2 I_{0}(b \omega) I_{1}(b \omega)-b \omega I_{1}^{2}(b \omega)}
$$

$$
\begin{aligned}
& S_{3}(\omega)=\frac{A_{n}^{(1)}(\omega)-b \omega I_{0}(b \omega) S_{1}(\omega)}{\omega I_{1}(b \omega)} \\
& S_{4}(\omega)=\frac{A_{n}^{(2)}(\omega)-b \omega I_{0}(b \omega) S_{2}(\omega)}{\omega I_{1}(b \omega)}
\end{aligned}
$$

where

$$
A_{n}^{(1)}(\omega)=-(-1)^{n / 2} \frac{2 \omega^{n}}{\pi n !} K_{1}(b \omega)
$$

$$
\begin{aligned}
A_{n}^{(2)}(\omega)=(-1)^{n / 2} \frac{2 \omega^{n-2}}{\pi n !}[(n-2) & (n-3) K_{1}(b \omega) \\
& \left.-(2 n-3) b \omega K_{0}(b \omega)\right]
\end{aligned}
$$

$$
A_{n}^{(3)}(\omega)=(-1)^{n / 2} \frac{2 \omega^{n}}{\pi n !} K_{0}(b \omega)
$$

$A_{n}^{(4)}(\omega)=(-1)^{n / 2} \frac{2 \omega^{n-2}}{\pi n !}\left[(2 n-3) b \omega K_{1}(b \omega)\right.$

$$
\left.-n(n-1) K_{0}(b \omega)\right]
$$

Manuscript received Dec. 2, 2004, and revision received Jan. 24, 2005. 